\title{
Direct synthesis procedure for sub-harmonic stub band-pass and band-stop filters
}

\author{
T. Stander, S. Sinha \\ Carl and Emily Fuchs Institute for Microelectronics \\ Department of Electrical, Electronic and Computer Engineering \\ University of Pretoria \\ c/o Lynnwood and University Road \\ Pretoria, 0002 \\ South Africa \\ E-mail: tinus.stander@up.ac.za
}

\begin{abstract}
A non-iterative synthesis procedure for sub-harmonic stub (SHS) bandpass and band-stop filters is presented. The method ensures better agreement between the synthesized filter response and the original prototype function than was possible previously without iterative techniques. The procedure is illustrated for two L-band microstrip filters, with good agreement between modeled and measured responses.
\end{abstract}

Keywords: Microstrip filters, transmission line filters, filter synthesis

Introduction: With the advent of miniaturized satellite systems such as the CubeSat standard, multi-layer and compact system-in-package (SIP) microwave front-ends require filter topologies with minimum filter order and complexity, yet increasing selectivity. Finite transmission zeros in filters are used both for increasing pass-band selectivity [1] and creating wide stop-bands [2]. These zeros are typically realized with cross-coupled elements [3, 4], multiple mode resonators [5] or multiple transmission paths [6], all of which add to the filter's order, size and synthesis complexity. 
A simple transmission line topology for realizing transmission zeros in both band-stop and band-pass filters was proposed [7, 8] whereby a conventional stub filter's shunt stubs were elongated to $2 l_{1}$ and $4 l_{1}$ to form sub-harmonic stubs (SHS), where $I_{1}$ is the conventional $\lambda_{0} / 4$ length of a stub generated by Richard's transform [1] at a given stop-band or pass-band center frequency $f_{0}$. In both cases, the authors relied on iteratively selected, rather than synthesized, values of impedance for the elongated stubs. This paper presents a synthesis procedure whereby an arbitrary frequency may be chosen to match the responses of the SHS filter and the original low-pass prototype response, thereby establishing a non-iterative synthesis method for this class of filters. This agreement may be used to preserve pass-band reflection, width or any other chosen parameter through the synthesis process. The process is demonstrated on both band-pass and band-stop filters.

Band-pass synthesis procedure: The filter synthesis starts as per the traditional method for synthesizing short- and open-circuited stub filters [1]. The parameters selected for the Chebyshev filter were $N=3$ with $325 \mathrm{MHz}$ transmission bandwidth around $f_{0}=1 \mathrm{GHz}$ and $0.5 \mathrm{~dB}$ pass-band transmission ripple. Using the transform

$$
\Omega_{\mathrm{c}}=\tan \left(\left(\pi \mathrm{f}_{\mathrm{c}}\right) /\left(2 \mathrm{f}_{0}\right)\right)
$$

the cut-off in the low-pass prototype is calculated as $3.832 \mathrm{Rad} / \mathrm{s}$, leading (though low-pass to high-pass transformation and Richard's transforms) to the generalized circuit in Fig. 1 with block "A" representing a short circuit and "B" an open circuit termination. The response of the prototype function is marked as trace $-\Delta-$ in Fig. 2 with synthesized values of $Z_{2}=219.8 \Omega, Z_{1,3}=12.65 \Omega$ and $f_{1,2,3}=1 \mathrm{GHz}$.

To realize the finite transmission zeros in the stop-band the short-circuited terminations of the shunt stubs (marked "A" in Fig. 1) are replaced by open-circuited 
terminations, and the stubs $Z_{1}$ and $Z_{3}$ elongated. The impedance value of these SHS lines are calculated by considering the input impedance of the original shunt inductance $L$ in the low-pass prototype

$$
Z_{\text {in(L) }}=j \Omega_{m} L
$$

at any given Richards' variable value $\Omega_{\mathrm{m}}$, which transforms uniquely to a given real frequency $f_{m}$. The input impedance of the open-circuit stub, at this frequency, is calculated as

$$
Z_{\text {in(Stub) }}=-j Z_{1} \cot \left(\left(\pi f_{m}\right) /\left(2 f_{z}\right)\right)
$$

where $f_{z}$ is the frequency where the stub's length is equal to $\lambda_{z} / 4$. A frequency $f_{m}$ of exact correspondence may now be chosen, which will produce a unique impedance value $Z_{l}$ for the stub by equating Eq. (2) and Eq. (3). The difference in choosing $f_{m}$ in the band $(0.9 \mathrm{GHz})$ or at the band edge $(0.837 \mathrm{GHz})$ is shown in Fig. 2. It is observed that the in-band choice at $0.9 \mathrm{GHz}$ achieves a better transmission band approximation to the original Chebyshev prototype function, and is chosen for manufacturing. The value of $Z_{2}$ remains unchanged from the initial synthesis, with $Z_{2}$ $=79 \Omega, Z_{3}=27 \Omega, f_{1}=0.25 \mathrm{GHz}$ and $f_{3}=0.5 \mathrm{GHz}$. To avoid having to manufacture an impractically high $219.8 \Omega$ stub impedance or uniplanar short-circuited series stubs [9], the central open-circuit stub is divided into a cascade combination of two $109.9 \Omega$ stubs (Fig. 3, with "A" representing open-circuited terminations). This split does, however, introduce a short but unavoidable section of transmission line $Z_{4}$.

Band-stop synthesis procedure: Initial synthesis of the filter is identical to the method illustrated for band-pass filters, with the parameters of the Chebyshev prototype function chosen as $N=3,0.5 \mathrm{~dB}$ pass-band transmission ripple and cut-off frequency 
$f_{c}=0.163 \mathrm{GHz}$. The transformed Richards variable is calculated in Eq. (1) as 0.261 Rad/s, leading to the same generalized circuit shown in Fig. 1. In the case of the band-stop filter, "A" represents an open-circuited termination, whilst "B" represents a short circuit. The synthesized element values are $Z_{2}=219.8 \Omega, Z_{1,3}=12.65 \Omega$ and $f_{1,2,3}=1 \mathrm{GHz}$. The circuit response is shown as the trace marked $-\Delta-$ in Fig. 4 .

In the band-stop case, instead of calculating the appropriate impedance from a shunt inductor as in Eq. (2), the impedance of the two elongated stubs are calculated from the equivalent input impedance of a shunt capacitance $C$ in the lumped element lowpass prototype

$$
Z_{\text {in }(C)}=-j /\left(\Omega_{m} C\right)
$$

at a given Richards' variable value $\Omega_{m}$, which transforms to a given real frequency $f_{m}$. This impedance is again equated to that of an an open-circuited shunt stub as in Eq. (3), yielding a unique impedance value for a given $f_{m}$. The result of choosing $f_{m}$ at the band center $(0.1 \mathrm{GHz})$ and band edge $(0.163 \mathrm{GHz})$ are illustrated in Fig. 4. The former achieves a better approximation to the original Chebyshev prototype function in-band, and is selected for manufacturing. The value of $Z_{2}$ remains unchanged from the initial synthesis, with $Z_{1}=58 \Omega, Z_{3}=26 \Omega, f_{1}=0.25 \mathrm{GHz}$ and $f_{3}=0.5 \mathrm{GHz}$. As with the band-pass filter, the $219.8 \Omega$ stub is replaced by two $109.9 \Omega$ stubs separated by a short section of transmission line, producing the same circuit model as in Fig. 3 but with "A" representing a short-circuited termination in the case of a band-stop filter.

Simulation and measurement results: Both filters were realized on two layers of $32 \mathrm{mil}$ Rogers RO4003C, as dimensioned in Fig. 5 and pictured in Fig. 6. For both the bandpass and band-stop filters, the light shaded trace is the filter's top signal layer forming 
the two series stubs $Z_{2}$ in Fig. 3. In the band-stop filter's case, the top strip is terminated with a via down to the lower signal layer, whilst in the band-pass filter these vias and via pads are omitted.

The band-pass filter is dimensioned (Fig. 5 ) as $w_{0}=1.5, w_{1}=1.86, w_{2}=0.86, w_{3}=$ $6.29, I_{1}=169.01, I_{2}=91.86, I_{3}=82.7$ and $s=9.44$. The previously noted $Z_{4}$ is created by the physical separation between the two stubs on the upper signal layer (dimensioned $s$ in Fig. 5), and causes some discrepancy between the original circuit response and the simulated response. This effect is modeled by a line of length $\lambda / 4$ at $0.8 \mathrm{GHz}$ and impedance $109.93 \Omega$. The measured results (Fig. 7) were found to be in good agreement with the simulated response. The final filter features $0.6 \mathrm{~dB}$ mid-band insertion loss and $-10 \mathrm{~dB}$ input match over a $280 \mathrm{MHz}$ band centered at 1 $\mathrm{GHz}$, with minimum out-of-band attenuation of $-22 \mathrm{~dB}$ from $\mathrm{DC}$ to $4 \mathrm{GHz}$.

The band-stop filter is dimensioned (in $\mathrm{mm}$, as shown in Fig. 5) as $d_{\text {via }}=0.25 \mathrm{~mm}, w_{0}$ $=1.5, w_{1}=1.12, w_{2}=0.4, w_{3}=4.15, l_{1}=164.25, I_{2}=90.4, l_{3}=80.6, d_{p a d}=0.5$ and $s$ $=2.63$. The responses in Fig. 7 show a pole created near $f_{0}$ due to the presence of the transmission line of length $s$ on the upper signal layer. This is replicated in the circuit model (Fig. 8) with a transmission line of $Z_{4}=109.9 \Omega$ and $f_{4}=2.3 \mathrm{GHz}$. Despite this unwanted circuit element, the filter exhibits $-20 \mathrm{~dB}$ attenuation over the stop-band and below $0.1 \mathrm{~dB}$ pass-band ripples on either side of the stop-band, with the evenly spaced finite transmission zeros clearly visible.

Conclusion: A non-iterative synthesis process has been demonstrated for subharmonic stub band-stop and band-pass filters. The method has been demonstrated to produce filters with realizable impedance values that follow the input reflection response of the original prototype function better than was previously possible. The method suffers from some performance degradation due to unwanted additional 
transmission lines in physical realization, but good agreement between circuit simulation and measured results are still obtained if these lines are taken into account in modeling.

Acknowledgments: The authors wish to thank CST AG for the use of CST Microwave Studio 2012 under the educational license provided, as well as Denel Dynamics (a division of Denel (SOC) Ltd.) for measurement facilities. This work was supported by the National Research Foundation of South Africa (Grant ID\# 73666).

\section{References}

1 R. J. Cameron, R. R. Mansour, and C. M. Kudsia, Microwave filters for communication systems, John Wiley \& Sons Inc., 2007.

2 M. Sanchez-Soriano, G. Torregrosa-Penalva, and E. Bronchalo, Compact wideband bandstop filter with four transmission zeros, IEEE Microw Wirel Compon Lett 20 (2010), 313-315.

3 V. Radonic, and V. Crnojevic-Bengin, Cross-coupled microstrip filter using grounded patch resonators, Proc 41st European Microwave Conf (2011), 763-766.

4 A. B. Guntupalli, V. K. Velidi, and S. Sanyal, Compact dual wide bandstop filter, Proc Applied Electromagnetics Conf (2009), 1-4.

5 B. Li, X. Wu, and W. Wu, A miniaturized branch-line coupler with wideband harmonics suppression, Progress in Electromagnetics Research 17 (2010), 181-189. 
6 K. Divyabramham, M. K. Mandal, and S. Sanyal, Sharp-rejection wideband bandstop filters, IEEE Microw Wirel Compon Lett 18 (2008), 662-664.

7 J. A. G. Malherbe, Wideband bandstop filter with sub-harmonic stubs, Electron Lett 47 (2011), 604-605.

8 J. A. G. Malherbe, Pseudo-elliptic bandpass filter with subharmonic stubs, Electron Lett 47 (2011), 196-198.

9 T. M. Weller, K. J. Herrick, and L. P. B. Katehi, Bandstop series stubs for coplanar waveguide on GaAs, Electron Lett 33 (1997), 684 - 685. 
Figure captions

Fig. 1 Generalized circuit model of initial SHS filters.

Fig. 2 Circuit model responses of band-pass filter

$-\Delta-\quad$ Initial stub filter

-O- $\quad$ SHS filter, $f_{m}=0.837 \mathrm{GHz}$

$-\mathrm{x}-\quad$ SHS filter, $f_{m}=0.9 \mathrm{GHz}$

Fig. 3 Generalized expanded circuit model of SHS filters.

Fig. 4 Circuit model responses of band-stop filters

$-\Delta-\quad$ Initial stub filter

-O- $\quad$ SHS filter, $f_{m}=0.1 \mathrm{GHz}$

$-x-\quad$ SHS filter, $f_{m}=0.163 \mathrm{GHz}$

Fig. 5 Microstrip layout of SHS filters. Shaded area is upper signal layer, white outline is lower signal layer.

Fig. 6 Photographs of manufactured band-pass (top) and band-stop (bottom) filters.

Fig. 7 Simulated and measured SHS band-pass filter responses compared to circuit response.

$-\Delta-\quad$ Circuit model response, $Z_{4}$ added.

-O- Simulated response.

$-x-\quad$ Measured response.

Fig. 8 Simulated and measured responses of SHS band-stop filter compared to circuit response.

$-\Delta-\quad$ Simulated response.

-O- Circuit model response, $Z_{4}$ added.

-x- Measured response. 
Figure 1

$Z_{2}, \Theta=90^{\circ} @ f_{2}$

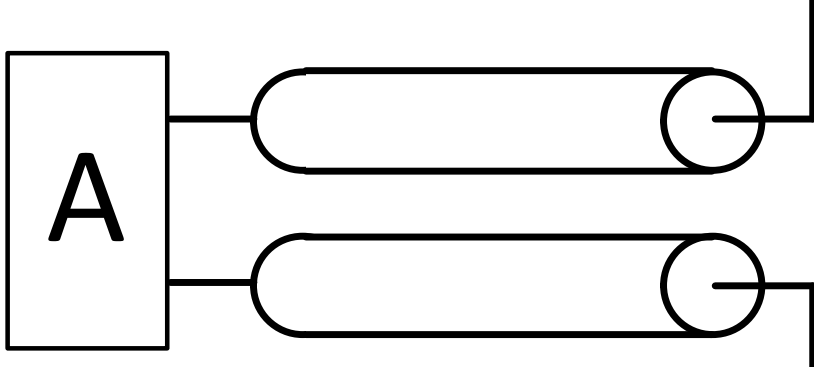

$-0$

A

$Z_{1}, \Theta=90^{\circ} @ f_{1}$

$Z_{3}, \Theta=90^{\circ} @ f_{3}$ 
Figure 2

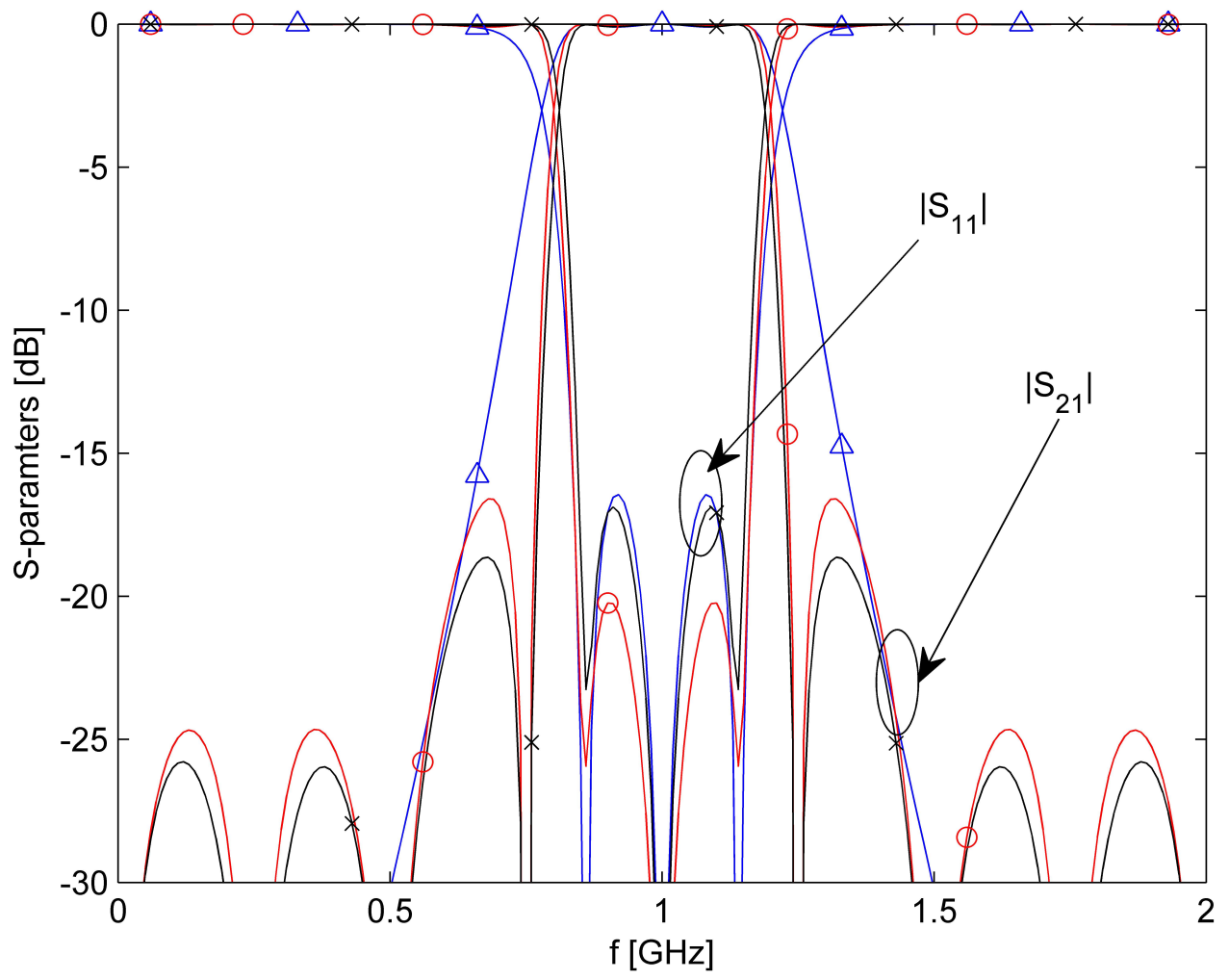




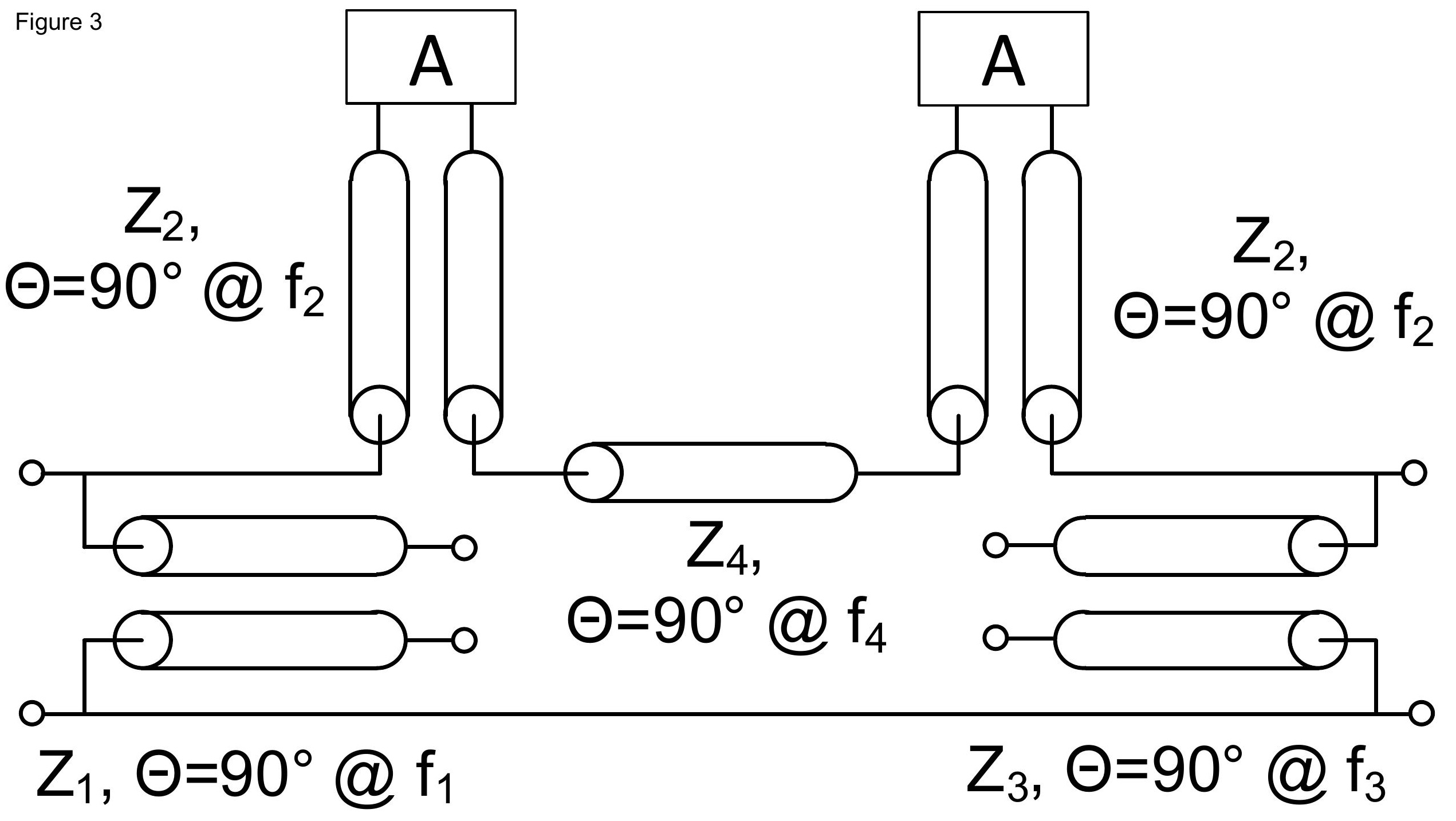


Figure 4

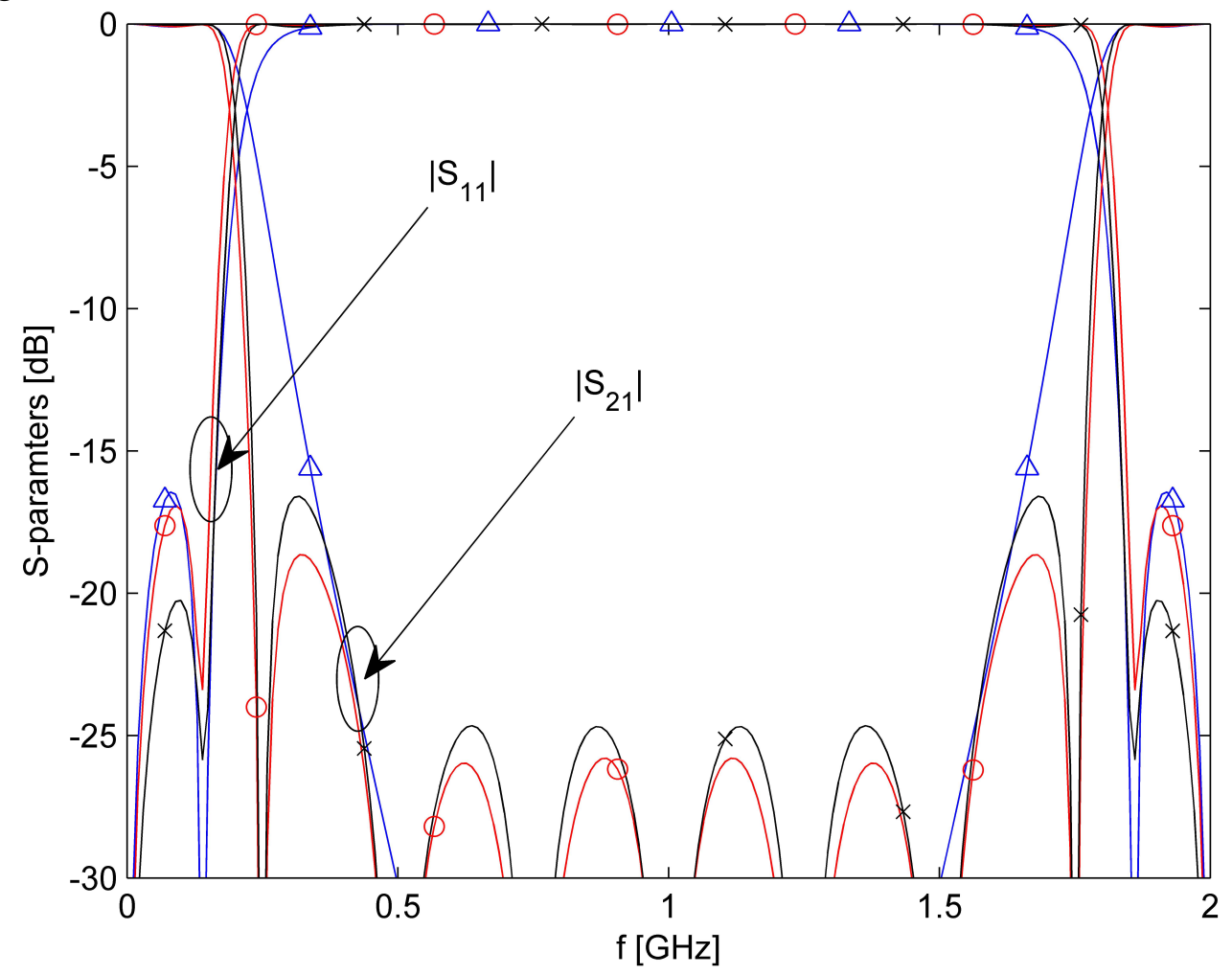


Microstrip port $1-\Delta_{\square}$

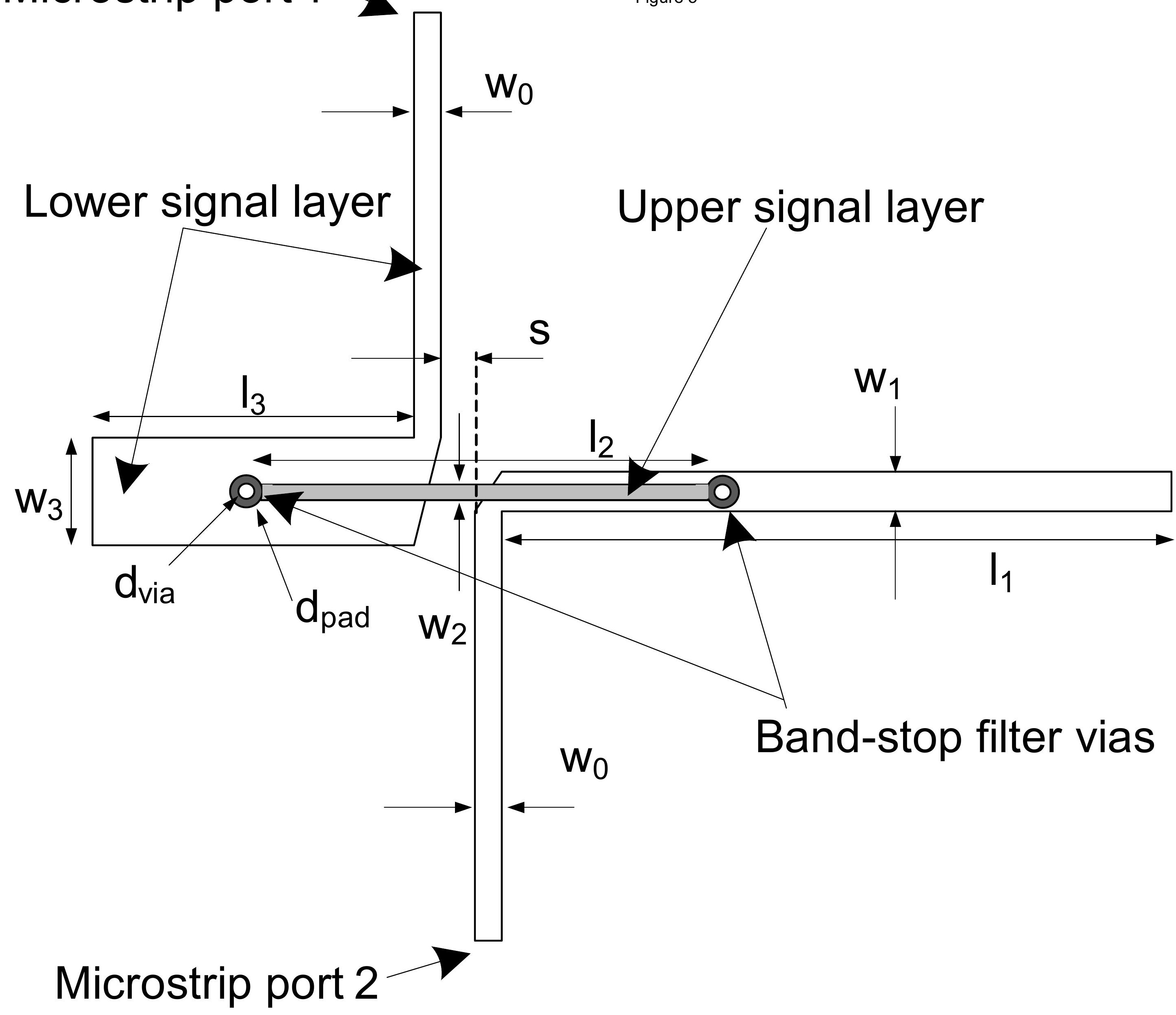


Figure 6 
Figure 7

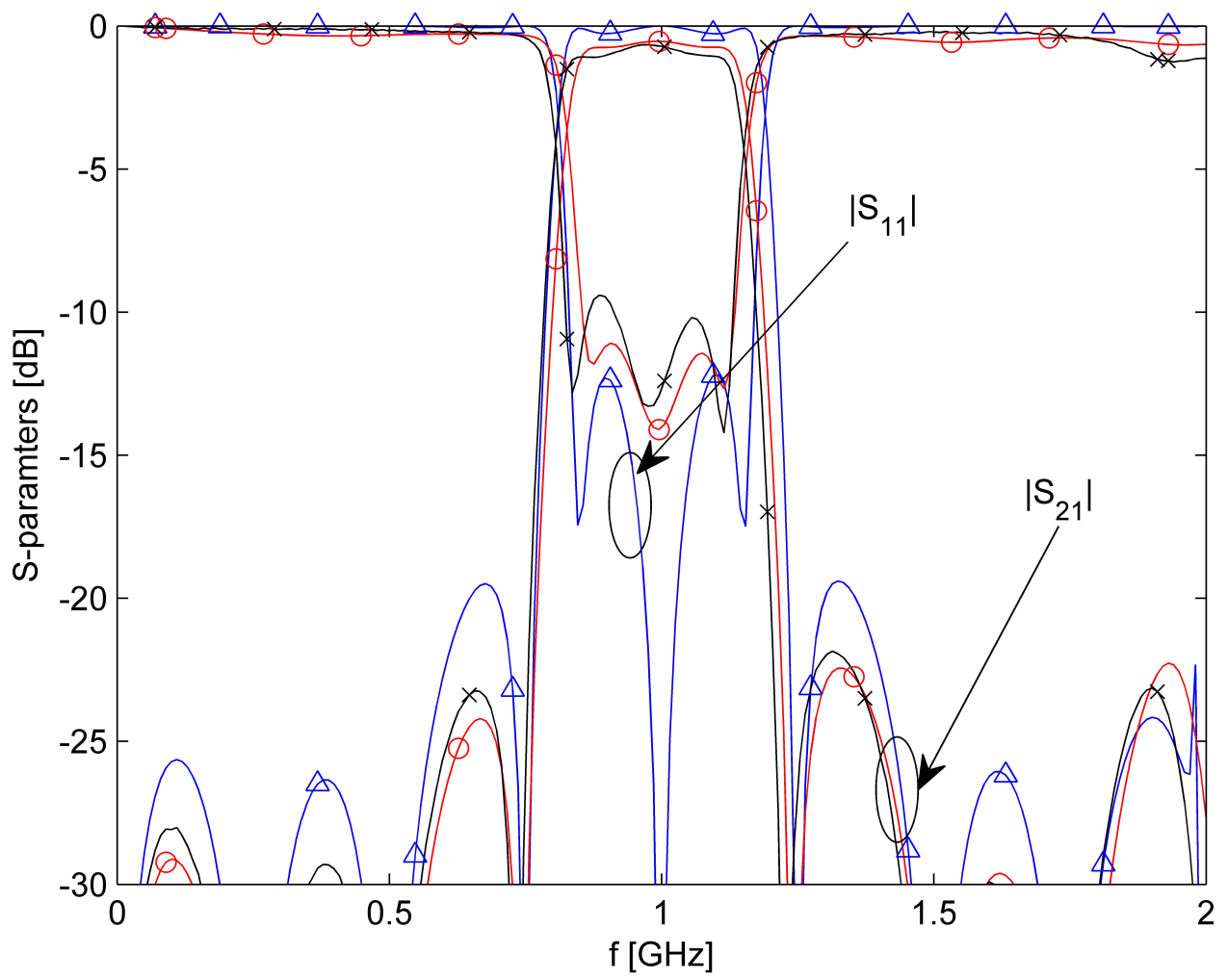


Figure 8

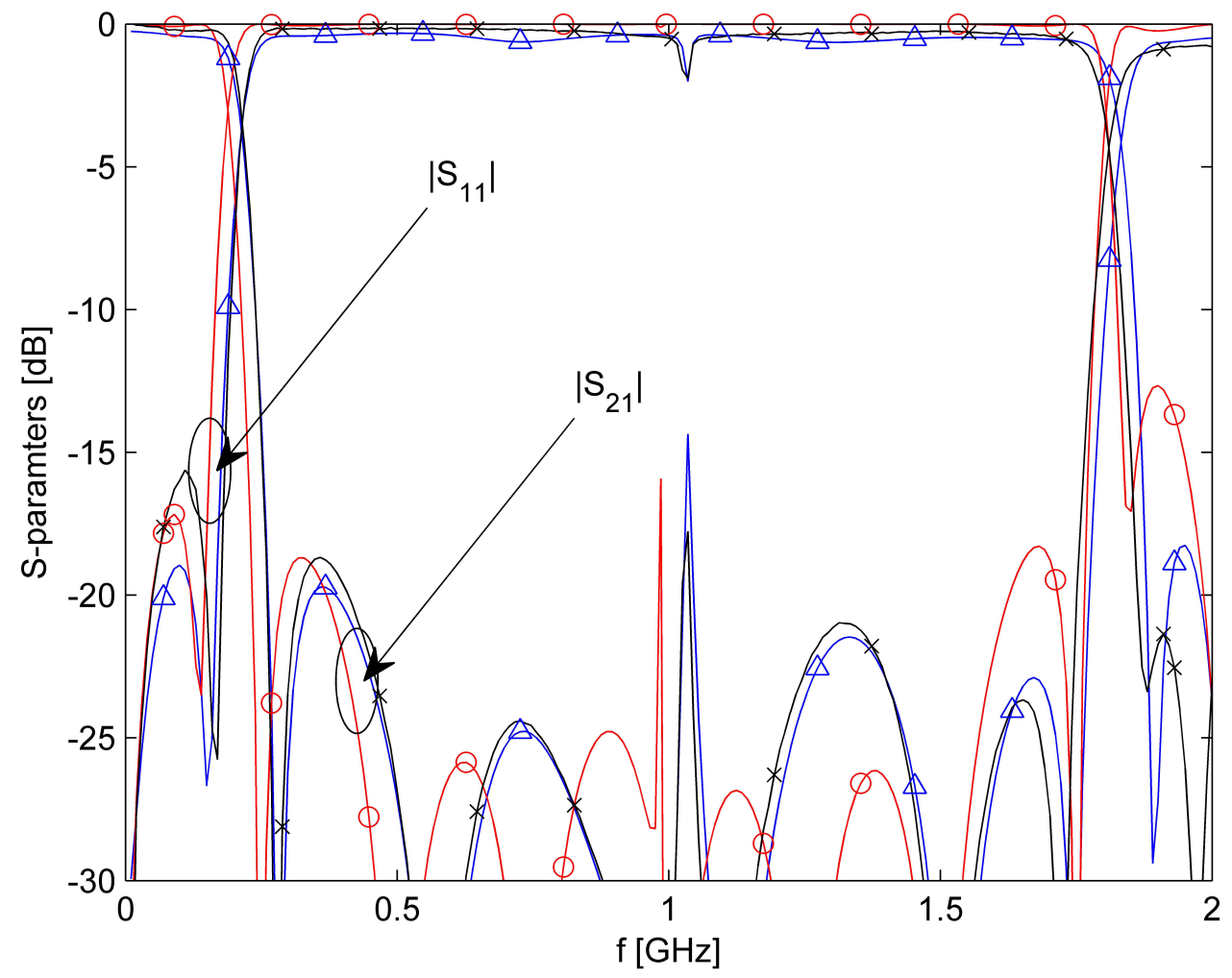

